\title{
The novel roles of circRNAs in human cancer
}

\author{
Qingfeng Shang ${ }^{1,2+}$, Zhi Yang ${ }^{1,2,3 \dagger}$, Renbing Jia ${ }^{1,2^{*}}$ and Shengfang Ge ${ }^{1,2^{*}}$
}

\begin{abstract}
Covalently closed single-stranded circular RNAs (circRNAs) consist of introns or exons and are widely present in eukaryotic cells. CircRNAs generally have low expression levels and relatively stable structures compared with messenger RNAs (mRNAs), most of which are located in the cytoplasm and often act in cell type and tissue-specific manners, indicating that they may serve as novel biomarkers. In recent years, circRNAs have gradually become a hotspot in the field of RNA and cancer research, but the functions of most circRNAs have not yet been discovered. Known circRNAs can affect the biogenesis of cancers in diverse ways, such as functioning as a microRNA (miRNA) sponges, combining with RNA binding proteins (RBPs), working as a transcription factor and translation of proteins. In this review, we summarize the characteristics and types of circRNAs, introduce the biogenesis of circRNAs, discuss the emerging functions and databases on circRNAs and present the current challenges of circRNAs studies.
\end{abstract}

Keywords: CircRNAs, Cancer, Sponge, Translation, Database

\section{Background}

In the past few decades, the field of RNA, especially the non-coding RNA(ncRNA)field,has benefitted from the rapid development and application of high-throughput RNA sequencing (RNA-seq) technology [1]. The majority of RNA species in eukaryotic cells is comprised of ncRNA rather than messenger RNA (mRNA), and studies have shown that these ncRNAs play a vital role in physiological and developmental processes. In previous studies, scientists mainly focused on linear ncRNAs, such as long non-coding RNAs (lncRNAs) and microRNAs (miRNAs), indicating that these linear ncRNAs have multiple functions in physiological and pathological processes. As a kind of unique circular ncRNA, circular RNAs (circRNAs) have been previously considered accidental by-products or 'splicing noise' with low abundance and little functional potential, resulting from errors during post-transcriptional processing [2]. The first circRNAs were found in the Sendai virus and plant-infected viroids in 1976 by Kolakofsky and Sanger, respectively [3, 4]. Subsequently, only a few circRNAs

\footnotetext{
*Correspondence: renbingjia@sjtu.edu.cn; geshengfang@sjtu.edu.cn

${ }^{+}$Qingfeng Shang and Zhi Yang contributed equally to this work.

${ }^{1}$ Department of Ophthalmology, Ninth People's Hospital, Shanghai Jiao Tong

University School of Medicine, No. 12, Lane 833, Zhizaoju Road, Huangpu

District, Shanghai 200001, China

Full list of author information is available at the end of the article
}

with or without biological functions were discovered in eukaryotes [5-8]. Now, however, due to the widespread application of new technologies, circRNAs have been recognized and taken seriously in various biological fields. RNA-seq and bioinformatic analysis have proven that thousands of circRNAs are abundant in the brain $[9,10]$, and recent studies have experimentally confirmed the significant biological functions of circRNAs, especially in the field of cancer [11].

\section{Characteristics of circRNAs}

CircRNAs are covalently closed, single-stranded circular transcripts with no $5^{\prime}$ caps and $3^{\prime}$ poly(A) tails; this structural characteristic makes circRNAs resistant to the digestion of ribonucleases, such as RNase $R$ and exonuclease, and confers a longer half-life than that of linear mRNAs [12]. In addition, most circRNAs are evolutionarily conserved across species [13]. CircRNAs are often expressed at low levels [14-16], implying the possibility that they may act as 'splicing noise' with little functional potential. However, multiple circRNAs detected by deep sequencing have been experimentally shown to be expressed more abundantly than their linear counterparts, sometimes even more than 10 times $[16,17]$. Most circRNAs are often located in the cytoplasm, consisting of exons, while a small part of 
circRNAs consisting of introns are located in the nucleus [18], and they are generally expressed in cell type-specific and tissue-specific manners [19].

\section{Biogenesis of circRNAs}

CircRNAs are produced from precursor mRNA (pre-mRNA), and they are transcribed by RNA polymerase II [20]. The currently discovered circRNAs can be simply sorted into three types according to their different composition and cycling mechanisms: exonic circRNAs, intronic circRNAs and exon-intron circRNAs (EIciRNA). At present, the maturation mechanism of circRNAs is not fully understood. It is inferred that exonic circular RNA is formed by backsplicing [1]. There are currently three hypothetical models explaining the formation of exonic circRNAs: lariat-driven circularization, intron-pairing-driven circularization and RNA binding protein (RBP) mediated circularization [14] (Fig. 1). In the process of forming exonic circRNAs, partial RNA folding occurs during pre-mRNA transcription, and the exon skips along with folding of the RNA. These structural changes result in the formation of specific regions, called lariat structures, in which originally non-adjacent exons become close to each other along with their introns. CircRNA is then formed after the intron sequence is removed by splicing within the lariat structure. This model is defined as lariat-driven circularization. Due to the presence of reverse complement sequences in introns on both sides of pre-mRNA, the complementary pairing of introns on both sides mediates the formation of circRNA. This model is defined as intron-pairing-driven circularization. Additionally, some RNA binding proteins are found to be critical in the formation of circRNAs. The highly conserved RNA-editing enzyme ADAR can bind double-stranded RNAs by targeting double-stranded ALU repeats in human cells [21-23]. ADAR1 antagonizes circRNA biogenesis through A-to-I editing of intron pairs flanking circularized exons, thus diminishing the complementarity and stability of these intron pair interactions [9, 23, 24]. DHX9, an abundant nuclear RNA helicase, has a unique domain organization that resembles ADAR. Silencing DHX9 leads to increased circRNA production through unwinding RNA pairs flanking circularized exons in general. Interestingly, there is a conserved RNA-independent interaction between ADAR (p150) and DHX9 in mouse and human cells, and co-depletion of DHX9 and ADAR can even promote more circRNA production [25]. Errichelli et al. revealed that FUS regulates circRNA formation by binding introns flanking back-splicing junctions in mouse embryonic stem cell (ESC)-derived motor neurons [26]. Heterogeneous nuclear ribonucleoprotein L (HNRNPL) was found to directly regulate the alternative splicing of multiple RNAs. A recent study found that

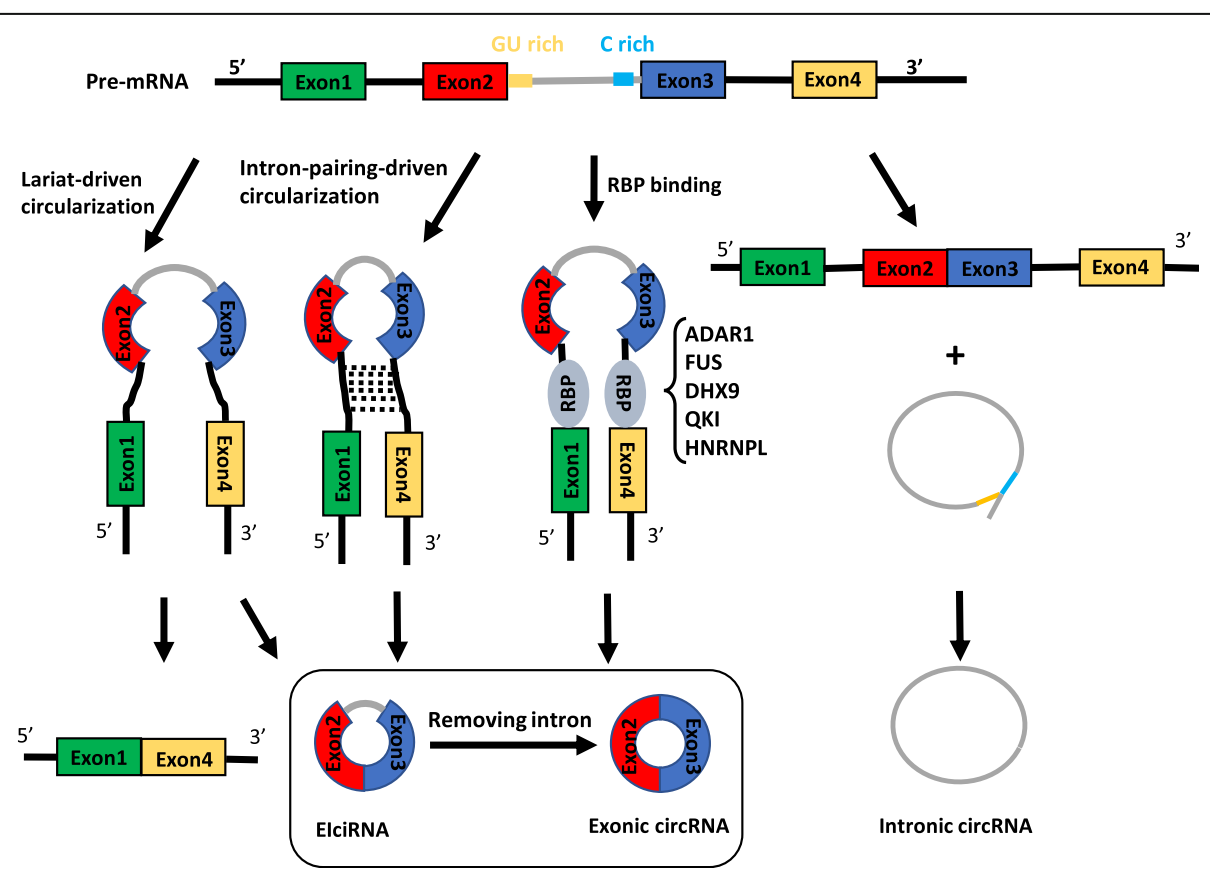

Fig. 1 Biogenesis of circRNAs. Different types of circRNAs are generated via different mechanisms. In lariat-driven circularization, the exon skips along with RNA folding, and exonic circRNAs or ElciRNAs are formed with or without removing introns. In intron-pairing-driven circularization, the formation of exonic circRNAs or ElciRNAs are regulated by base pairing in introns on both sides of pre-mRNA. RBPs bind to the specific sequence of introns, which affect the biogenesis of circRNA. In the formation of intronic circRNAs, the intron lariat is formed with the combination of conserved motifs located at upstream and downstream of introns 
HNRNPL was also involved in the regulation of circRNAs in prostate cancer [27]. Quaking (QKI), as a kind of alternative splicing factor, promotes circRNA generation by binding to its intronic binding motifs. The addition of a synthetic QKI binding motif into introns was sufficient to induce de novo circRNA formation [28]. With respect to intronic circRNAs, it is currently believed that some introns form lariat structures during splicing, but most will be degraded rapidly by debranching, and only some containing the essential nucleic acid sequences, such as a seven nucleotide GU-rich motif near the $5^{\prime}$ splicing site and an eleven nucleotide $\mathrm{C}$-rich motif near the branch point, will not be debranched after splicing, thus forming intronic circRNA [29]. EIciRNAs are composed of exons and introns. In the formation of exonic circRNAs, introns that surround the exons are usually spliced out, however, in some cases, they are retained and are thus named EIciRNA [30].

\section{Function of circRNAs \\ circRNA can act as ceRNA}

The ceRNA(competing endogenous RNA) hypothesis indicates that miRNA can affect mRNA stability and transcription at the post-transcriptional level by binding to target genes; thus, RNA can also affect miRNAs [31]. This hypothesis mainly involves three kinds of RNAs, including mRNA, transcribed pseudogenes and lncRNA [32], and now circRNA, which has followed lncRNA in becoming a new research hotspot among the ceRNA family.

Many circRNAs have different types and amounts of miRNA binding sites that can specifically bind to miRNAs, thereby reducing miRNA activity and upregulating the expression of miRNA-related target genes [33] (Fig. 2a). Researchers found that the circRNA ciRS-7 (circular RNA sponge for miR-7) has more than 60 conserved binding sites for miRNA-7 [34]. In human and mouse brain tissue, ciRS-7 acts as a molecular sponge of miR-7 and inhibits functions of miRNA, which in turn positively regulates miR-7 target genes. Recently, a study found that a long non-coding RNA Cyrano, a circRNA ciRS-7, and two microRNAs miR-671 and miR-7 form a regulatory network through sponge function in the mammalian brain [35]. This study reveals a molecular regulatory network composed entirely of non-coding RNAs and found that there may be unknown new mechanisms regulating the degradation of circRNA. Studies also revealed that circMTO1 was mainly located in the cytoplasm and that its overexpression could decrease cell proliferation and migration levels in vitro or in vivo by targeting miR- 9 and increasing p 21 expression, which is the target of miR-9 [36]. Several research groups found that a well-known circRNA, circHIPK3, derived from exon 2 of the HIPK3 gene, contains 1099 nucleotides and is abundant in multiple human tissues. In addition, circHIPK 3 acts as a sponge for a variety of miRNAs, including the miR-124, miR-558, miR-30 families, miR-7, miR-4288, miR-654, miR-193a, miR-379 and miR-29b [17, 37-47]. Other studies indicated that circRNA ITCH inhibits bladder cancer and glioma progression in the circITCH-miR-17/miR-224-p21/PTEN axis and circITH-miR-214-ITCH axis [48, 49]. In conclusion, these findings mentioned above support the idea that circRNAs acting as miRNA sponges may be a common phenomenon in cancer.

\section{CircRNAs act as transcriptional regulators}

Although multiple reports support the role of circRNAs as sponges for miRNAs, some scholars have indicated that some intronic circRNAs and EIciRNAs can regulate protein production by regulating gene expression in a transcriptional or post-transcriptional manner [1, 14] (Fig. 2b). EIciRNA, such as EIciPAIP2 and EIciEIF3J, is mainly located in the nucleus and it can interact with the U1 small nuclear ribonucleoprotein(snRNP) and then promote parental gene transcription through binding to RNA polymerase II (RNA pol II) [30]. In addition, one study found that the second exon of the splicing factor MBL can cyclize to form a circRNA, which competes with the linear splicing of pre-mRNA and affects the formation of linear RNA to regulate the expression of related genes [20].

\section{CircRNAs bind to proteins}

Just as linear RNAs have been revealed to bind to proteins, several studies have shown that certain circRNAs can work as protein decoys, which sequester proteins by harbouring binding sites for specific proteins and block protein activity by working as competing elements (Fig. 2c). For example, circRNA, such as ciRS-7 and SRY, can degrade Argonaute 2 (Ago2) protein and inhibit transcription of mRNA [34, 50]. Circ-Foxo3 was mainly located in the cytoplasm, and it can bind to p21 and CDK2 and induce cell cycle arrest via the formation of the circ-Foxo3-p21-CDK2 ternary complex [51]. In addition, studies have found that circ-Foxo3 can also interact with senescence-related proteins ID1 and E2F1 and tumour-related proteins HIF1 $\alpha$ and FAK [52]. Exonic circRNA cia-cGAS (circular RNA antagonist for cGAS) is formed by exons 4, 5 and 6 of D430042O09Rik gene transcripts and is mainly located in the nucleus. Cia-cGAS binds to cGAS protein and inactivates the enzymatic activity of cGAS in long term haematopoietic stem cells (LH-HSCs) [53].

\section{CircRNAs can be translated}

As non-coding RNAs, circRNAs were once considered to fail to translate via cap-dependent mechanisms due to 


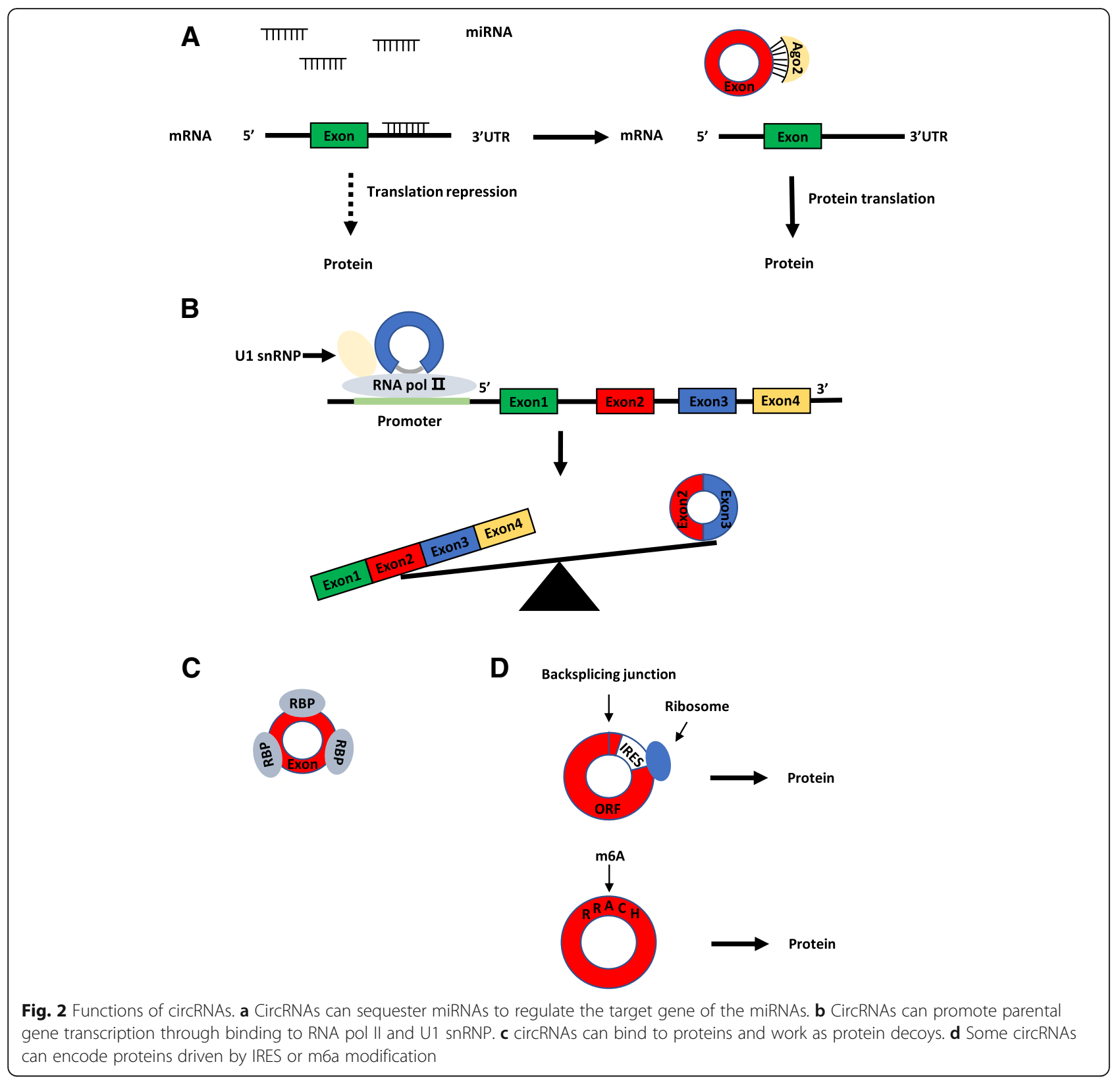

their lack of a 5'cap structure and a poly (A) tail. Additionally, previous polysome gradient analysis and ribosome profiling showed that most circRNAs are not associated with polysomes [14]. Therefore, circRNAs were generally considered to be non-coding RNAs due to lack of evidence for translation. However, most circRNAs consist of exons are mainly located in the cytoplasm. They may be related to the ribosome to some extent. Recently, strong evidence from many research groups has shown that circRNAs can encode proteins. Some researchers constructed a circular RNA containing an infinite reading frame, demonstrating that this type of circular RNA can mimic DNA rolling circle amplification (RCA) for RNA translation in prokaryotes [54].
Moreover, based on the discovery of the rolling circle translation of circular RNA in prokaryotes, researchers have speculated and verified that this model is equally applicable to the translation system of eukaryotic cells [55]. Based on high-throughput phenotypic screening, Legnini et al. identified circ-ZNF609, a functional circular RNA expressed in murine and human myoblasts that controls myoblast proliferation [56]. Circ-ZNF609 contains an open reading frame that passes the backsplicing junction and is associated with heavy polysomes, and it can be translated into a protein in a splicing-dependent and cap-independent manner. Moreover, Zhang et al. found that the circRNA circFBXW7, formed by exon 3 and exon 4 of the gene FBXW7, can be translated into a 
novel 21-kDa protein, which was termed FBXW7-185aa [57]. CircFBXW7 and FBXW7-185aa levels are reduced in glioma, and circFBXW7 expression was positively associated with glioblastoma patient overall survival. Moreover, FBXW7-185aa inhibited glioma proliferation and cell cycle acceleration in vitro and in vivo through reducing the half-life of $\mathrm{c}-\mathrm{Myc}$ by antagonizing USP28-induced c-Myc stabilization. Recently, Zhang et al. revealed that circ-SHPRH containing an open reading frame (ORF) driven by the internal ribosome entry site (IRES) can translate a functional protein, named SHPRH-146aa, in glioma [58]. Interestingly, the translation pattern of circ-SHPRH is quite similar to that of circular RNA of the virusoid associated with rice yellow mottle virus by using overlapping genetic codes to generate a 'UGA' stop codon. CircRNA is not easily digested by ribonuclease and is more stable than linear RNA due to its natural structural properties. Therefore, by constructing engineered exogenous circRNA, cyclizing mRNA can effectively solve the problem of the short half-life of linear mRNA, thereby expressing proteins efficiently and permanently in eukaryotic cells [59].

Interestingly, the translation of circRNAs can be mediated not only by IRES but N6-methyladenosine (m6a) can also drive the translation of circular RNA (Fig. 2d). Yang et al. found that approximately 13\% of the total circRNA had a m6A modification, that the circRNA carrying the m6A modified motif, the RRACH fragment $(\mathrm{R}=\mathrm{A}$ or $\mathrm{G} ; \mathrm{H}=\mathrm{A}, \mathrm{U}$ or $\mathrm{C})$ can translate the polypeptide intracellularly, and that the translation efficiency of the circRNA is affected by its m6A modification level [60].

\section{Databases of circRNAs}

With the development of circRNA field, many databases about circRNAs have been built to facilitates the analysis of circRNAs (Table 1). The Circbase, CIRC pedia v2 and Deepbase 2.0 databases contain numerous circRNAs about different species with detailed information [61-63]. The CircRNADb database contains genomic information, exon splicing, genome sequence, IRES, ORF and references about 32,914 human exonic circRNAs [64]. The Circnet and Starbase v2.0 databases offer researchers the circRNA-miRNA-gene regulatory networks with the application of bioinformatics $[65,66]$. The CSCD and CircInteractome databases can be used as tools to predict miRNA response elements(MRE)and RBP [67, 68]. The CirclncRNAnet database provides researchers an easy way to analysis sequencing results [69]. The ExoRBase database provides 58,330 circRNAs existed in human blood exosomes [70]. The circRNADisease database records experimentally verified circRNAs in mutiple diseases [71].

\section{CircRNAs in cancer}

To date, there has been multiple functional cancer-associated circRNAs screened and identified by different research groups (Table 2). These circRNAs play as oncogenes or tumor suppressors in multiple cancers and affect cancer phenotype via diverse ways.

\section{CircRNA in hepatocellular carcinoma}

Several studies have found that multiple circRNAs play a tumour suppressor role in hepatocellular carcinoma. CircMTO1 is underexpressed in hepatocellular carcinoma and affects the expression of downstream P21 protein by targeting miR-9 [36]. It was also revealed that circMTO1 is associated with a prognosis of hepatocellular carcinoma, indicating that circMTO1 may be a prognostic biomarker for hepatocellular carcinoma. Yu et al. found that the circRNA cSMARCA5 is downregulated in hepatocellular carcinoma and inhibits the proliferation and metastasis of hepatocellular carcinoma by sponging miR-181b-5p and miR-17-3p, thereby regulating TIMP3 expression [72]. In addition, the researchers also explored upstream of cSMARCA5 and found that the expression level of RNA helicase DHX9 was upregulated in hepatocellular carcinoma and that cSMARCA5 was negatively regulated. In addition, the study found that ZKSCAN1 mRNA and circular RNA circZKSCAN1 can both regulate the proliferation, invasion and migration of hepatocellular carcinoma through different pathways [73].

\section{CircRNA in breast cancer}

Derived from exon 4 and exon 5 of the CCNB1 gene, the circRNA circ-Ccnb1 was downregulated in breast cancer and mainly localized in the nucleus in breast cancer cells [74]. Based on an RNA pull-down assay to screen interacting proteins, it was found that circ-Ccnb1 can interact with p53 in p53 wild-type cells via H2AX but instead interacts with Bclaf1 in p53 mutant cells via $\mathrm{H} 2 \mathrm{AX}$, resulting in the induction of cell death in breast cancer. In triple-negative breast cancer, circGFRA1 acts as miR-34a ceRNA to regulate GFRA1 expression [75], while circEPSTI1 promotes triple-negative breast cancer cell proliferation through a novel pathway by sponging miR-4753 and miR-6809 and upregulating the oncogene BCL11A [76].

\section{CircRNA in leukaemia}

Multiple types of tumour cell genomes have chromosomal translocations and rearrangements, resulting in the formation of irrelevant gene recombination and fusion mRNAs that are ultimately translated into fusion proteins. Studies found that in acute promyelocytic leukaemia cells, the PML-RARa fusion gene can not only translate the fusion protein but also form a fusion 
Table 1 Database about circRNAs

\begin{tabular}{|c|c|c|c|}
\hline Database & Website & Function & Reference \\
\hline Circbase & http://www.circbase.org/ & $\begin{array}{l}\text { CircBase merged and unified data sets of } \\
\text { circRNAs and the evidence supporting their } \\
\text { expression, and also provided scripts to identify } \\
\text { known and novel circRNAs in sequencing data }\end{array}$ & [61] \\
\hline CIRCpedia v2 & http://www.picb.ac.cn/rnomics/circpedia & $\begin{array}{l}\text { CIRCpedia contained comprehensive circRNA } \\
\text { annotation from over } 180 \text { RNA-seq datasets } \\
\text { across six different species }\end{array}$ & [62] \\
\hline DeepBase v2.0 & http://rna.sysu.edu.cn/deepBase/ & $\begin{array}{l}\text { DeepBase v2.0 annotated 14,867 human } \\
\text { circRNAs }\end{array}$ & [63] \\
\hline CircRNADb & http://202.195.183.4:8000/circrnadb/circRNADb.php & $\begin{array}{l}\text { CircRNADb provided the detailed information of } \\
\text { the circRNA, including genomic information, } \\
\text { exon splicing, genome sequence, IRES, ORF and } \\
\text { references }\end{array}$ & [64] \\
\hline Circnet & http://circnet.mbc.nctu.edu.tw/ & $\begin{array}{l}\text { Circnet generated an integrated regulatory } \\
\text { network that illustrated the regulation between } \\
\text { circRNAs, miRNAs and genes }\end{array}$ & [65] \\
\hline Starbase v2.0 & http://starbase.sysu.edu.cn/ & $\begin{array}{l}\text { StarBase v2.0 systematically identified the RNA- } \\
\text { RNA and protein-RNA interaction networks from } \\
108 \text { CLIP-Seq data sets generated by } 37 \text { inde- } \\
\text { pendent studies }\end{array}$ & [66] \\
\hline CSCD & http://gb.whu.edu.cn/CSCD/ & $\begin{array}{l}\text { CSCD predicted the microRNA response } \\
\text { element sites, RNA binding protein sites and } \\
\text { potential open reading frames for each circRNA }\end{array}$ & [67] \\
\hline Circlnteractome & http://circinteractome.nia.nih.gov/ & $\begin{array}{l}\text { Circlnteractome searched public circRNA, } \\
\text { miRNA, and RBP databases to provide } \\
\text { bioinformatic analyses of binding sites on } \\
\text { circRNAs and additionally analyzes miRNA and } \\
\text { RBP sites on junction and junction-flanking } \\
\text { sequences }\end{array}$ & [68] \\
\hline CirclncRNAnet & http://app.cgu.edu.tw/circlnc/ & $\begin{array}{l}\text { CirclncRNAnet provided a "one-stop" resource } \\
\text { for in-depth analyses of ncRNA biology }\end{array}$ & [69] \\
\hline ExoRBase & http://www.exorbase.org/ & $\begin{array}{l}\text { ExoRBase provided annotation, expression level } \\
\text { and possible original tissues about } 58,330 \\
\text { circRNAs in human blood exosomes }\end{array}$ & [70] \\
\hline CircRNADisease & http://cgga.org.cn:9091/circRNADisease/ & $\begin{array}{l}\text { CircRNA Disease provided experimentally } \\
\text { supported circRNA and disease associations }\end{array}$ & [71] \\
\hline
\end{tabular}

circRNA f-circM9 [11]. A gain of function assay and loss of function assay have shown that f-circM9 plays a proto-oncogene role that contributes to cellular transformation in acute promyelocytic leukaemia. Additionally, f-circRNA conferred resistance to therapy in vivo and synergizes with other carcinogenic factors such as fusion proteins to promote cancer development.

\section{CircRNA in lung cancer}

Dai et al. found that circRNA circ0006916 acts as a tumour suppressor in lung cancer by binding to miR-522-3p and inhibiting PHLPP1 activity, thus inhibiting cell proliferation via slowing down the cell cycle process rather than by promoting apoptosis [77]. In addition, the RNA-binding protein TNRC6A binds to the flanking intron sequence of circ0006916 and regulates circRNA expression. This study successfully revealed the upstream mechanisms of circRNA circ0006916, and clearly validated the mechanism of circRNA in cell malignant transformation. Fusion genes not only exist in blood diseases but also in solid tumours. A novel circRNA, named as F-circEA, was generated from the EML4-ALK fusion gene by back-splicing in non-small cell lung cancer (NSCLC) [78]. F-circEA, independent of the EML4-ALK linear transcript and fusion protein, contributes to cancer progression by promoting cell migration and invasion. Notably, the evidence that F-circEA mainly exists in the plasma of EML4-ALK-positive NSCLC patients suggests that F-circEA could be a potentially novel biomarker for the diagnosis of EML4-ALK-positive NSCLC patients.

\section{CircRNA in glioma}

Yang et al. revealed that the endogenous circRNA circFBXW7, comprised of exon 3 and exon 4 of the FBXW7 gene, could encode a novel $21-\mathrm{kDa}$ protein, named FBXW7-185aa, driven by the IRES [57]. This protein can synergize with the protein encoded by FBXW7 to antagonize the stability of the proto-oncogene 


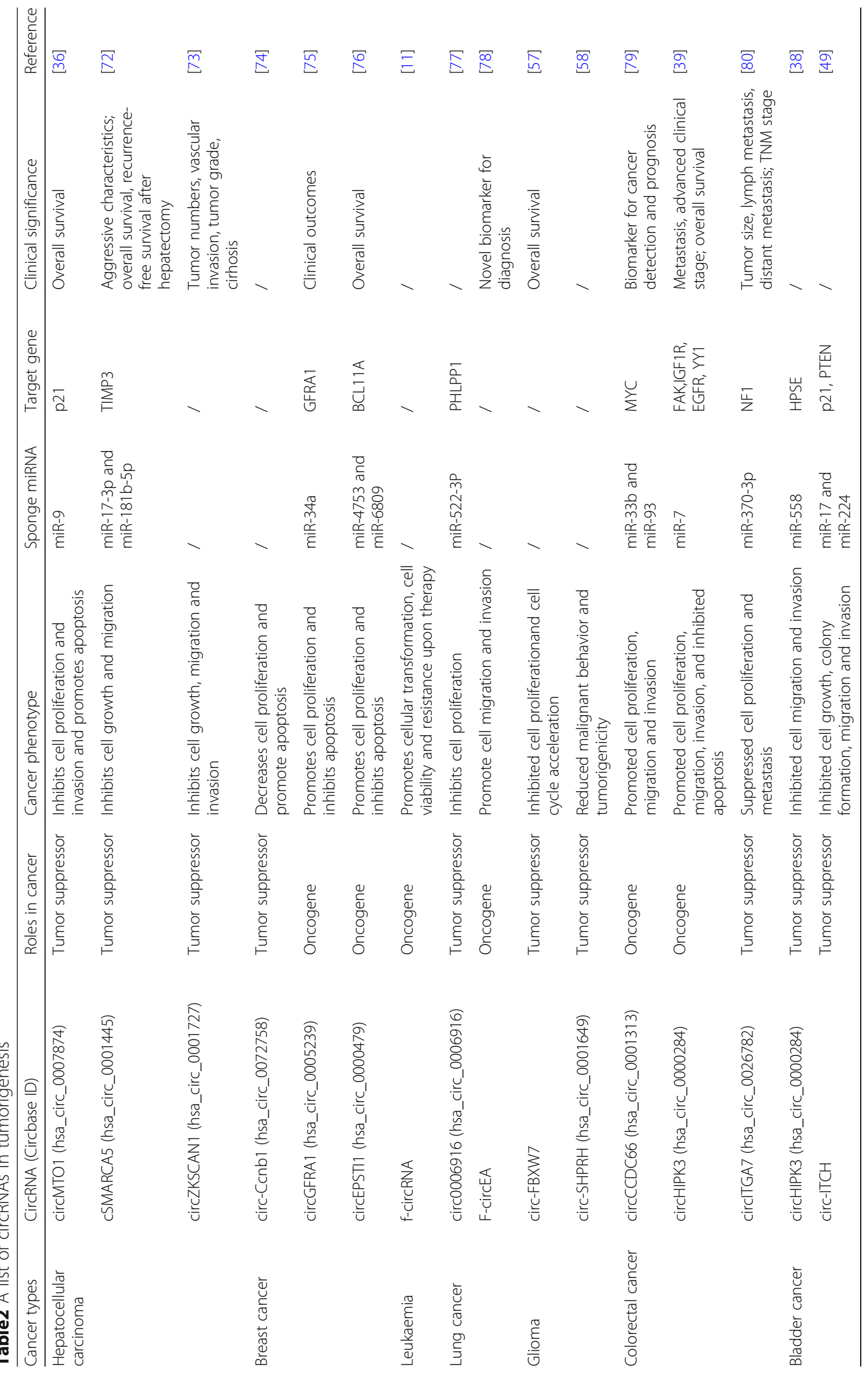


c-Myc and inhibit the progression of glioma. A study revealed the presence of the downregulated circRNA circ-SHPRH in gliomas, which translates a new $17 \mathrm{kDa}$ protein that named SHPRH-146aa by using overlapping genetic codes [58]. SHPRH-146aa protects full-length SHPRH from degradation by the ubiquitin proteasome. Stabilized SHPRH sequentially ubiquitinates proliferating cell nuclear antigen (PCNA) as an E3 ligase, leading to inhibited cell proliferation and tumourigenicity.

\section{CircRNA in colorectal cancer}

Recently, a study found that the circular RNA circCCDC66 is upregulated in a variety of tumour cells and is associated with poor prognosis of colorectal cancer [79]. CircCCDC66 regulates MYC expression by binding to miR-33b, miR-93, and miR-185. The upregulated circRNA circHIPK3 can promote proliferation, migration, invasion, and inhibit apoptosis in vitro and promote colorectal cancer growth and metastasis in vivo by increasing the expression levels of miR-7 targeting proto-oncogenes, such as FAK, IGF1R, EGFR, YY1 [39]. Researchers indicated a tumour suppressor role for circITGA7 and ITGA7 in colorectal cancer and revealed that circITGA7 inhibits proliferation and metastasis of colorectal cancer cells by suppressing the Ras signalling pathway and promoting the transcription of ITGA7 [80].

\section{CircRNA in bladder cancer}

Studies from $\mathrm{Li}$ et al. revealed that circRNA circHIPK3 was significantly downregulated in bladder cancer tissues and cell lines and was negatively correlated with bladder cancer grade, invasion as well as lymph node metastasis, respectively [38]. CircHIPK3 contains two critical binding sites for miR-558 and can suppress HPSE expression to inhibit migration, invasion and angiogenesis of bladder cancer cells. Yang et al. reported that circular RNA circ-ITCH regulates $\mathrm{p} 21$ and PTEN expression by sponging miR-17/miR-224, thus inducing cell cycle arrest and apoptosis [49].

\section{Challenges}

With the development of sequencing technology and bioinformatics, an increasing number of circRNAs are gradually being discovered and have become hotspots in the field of RNA. However, there are still quite a few challenges that need to be addressed. First, there is no uniform naming convention for circRNAs at present, which means researchers in different fields use different names when studying the same circRNA molecules, thus causing confusion. For example, cirs-7 indicates that this circular RNA acts as a sponge for miR-7, and CDR1as refers to a circRNA that is related to the CDR1 gene, but both actually refer to the same circRNA. Second, the current databases of circRNA are still not perfect.
Although researchers can predict the function of circRNA by using multiple databases, there is no circRNA database related to tumour prognosis, which makes it difficult to screen functional circRNA after RNA-seq. In addition, although sponging miRNAs is the most classical functional model of circRNA, whether this effect between miRNA and circRNA is universal is questioned. It has been reported that, based on the results of bioinformatics and experimental verification, the circRNA-miRNA functional model has a negative result [81]. Most of the circRNAs currently studied have fewer binding sites for miRNAs, compared with the relationship between CDR1as and miR-7, which greatly reduces the possibility of circRNAs binding to miRNAs. Therefore, the sponge functional model of circRNA and miRNA needs further research. Also, compared with other members of the RNA family, the mechanisms and functional models of the currently reported circRNAs are not diverse, which needs to be further explored, and there are problems for beginners in that the experimental techniques are relatively difficult.

\section{Conclusion}

The discovery of circRNA enriches cognition of biological evolution, increases understanding of RNA, and makes it a hotspot in the field of cancer research, which has given researchers a new direction in which to investigate the occurrence and development of tumours. Although knowledge of the precise mechanism of the formation, transportation and function of circRNAs in tumours is still very limited, with the application of new technologies and the constant efforts of scientists, the mystery of circRNA will eventually be solved.

\footnotetext{
Abbreviations

ADAR: Adenosine deaminase; Ago2: Argonaute 2; BCL11a: B cell CLL/ lymphoma 11A; Bclaf1: BCL2 associated transcription factor 1; CCNB1: Cyclin B1; ceRNA: Competing endogenous RNA; cia-cGAS: Circular RNA antagonist for CGAS; circRNAs: Circular RNAs; ciRS-7: Circular RNA sponge for miR-7; DHX9: DExH-box helicase 9; E2F1: E2F transcription factor 1; EGFR: Epidermal growth factor receptor; ElciRNA: Exon-intron circRNAs; ESC: Embryonic stem cell; FAK: Protein tyrosine kinase 2; FUS: FUS RNA binding protein; GFRA1: GDNF family receptor alpha 1; H2AX: H2A histone family member X; HIF1a: hypoxia inducible factor 1 subunit alpha; HNRNPL: Heterogeneous nuclear ribonucleoprotein L; HPSE: Heparanase; ID1: Inhibitor of DNA binding 1, HLH protein; IGF1R: Insulin like growth factor 1 receptor; IRES: Internal ribosome entry site; ITCH: Itchy E3 ubiquitin protein ligase; ITGA1: Integrin subunit alpha 1; LH-HSCs: Long term haematopoietic stem cells; IncRNA: Long non-coding RNAs; m6a: N6-methyladenosine; MBL: Muscleblind; miRNA: MicroRNA; MRE: MiRNA response elements; mRNAs: Messenger RNAs; ncRNA: Non-coding RNA; NSCLC: Non-small cell lung cancer; ORF: Open reading frame; p21: Cyclin dependent kinase inhibitor 1A; p53: Tumor protein p53; PCNA: Proliferating cell nuclear antigen; pre-mRNA: Precursor mRNA; PTEN: Phosphatase and tensin homolog; QKI: Quaking; RBPs: RNA binding proteins; RCA: Rolling circle amplification; RNA pol II: RNA polymerase II; RNA-seq: RNA sequencing; RRACH: m6A modified motif; snRNP: small nuclear ribonucleoprotein; SRY: Sex determining region Y; TIMP3: TIMP metallopeptidase inhibitor 3; YY1: YY1 transcription factor; ZKSCAN1: Zinc finger with KRAB and SCAN domains 1
} 


\section{Acknowledgements}

Not applicable.

\section{Funding}

This work was supported by the National Natural Science Foundation of China (Grant No. U1432117, 81772875, 81570884, 81770961), the Science and Technology Commission of Shanghai (17DZ2260100), the Innovation Fund for Translational Medicine (15ZH1005) and the ShuGuang Project of Shanghai Municipal Education Commission and Shanghai Education Development Foundation (14SG18).

\section{Availability of data and materials}

Not applicable.

\section{Authors' contributions}

OS and ZY collected the related literature and drafted the manuscript. RJ and SG participated in the design of the review and drafted the manuscript. All authors have read and approved the final manuscript.

\section{Ethics approval and consent to participate}

Not applicable.

\section{Consent for publication}

All authors have agreed on the contents of the manuscript.

\section{Competing interests}

The authors declare that they have no competing interests.

\section{Publisher's Note}

Springer Nature remains neutral with regard to jurisdictional claims in published maps and institutional affiliations.

\section{Author details}

${ }^{1}$ Department of Ophthalmology, Ninth People's Hospital, Shanghai Jiao Tong University School of Medicine, No. 12, Lane 833, Zhizaoju Road, Huangpu District, Shanghai 200001, China. ${ }^{2}$ Shanghai Key Laboratory of Orbital Diseases and Ocular Oncology, No. 12, Lane 833, Zhizaoju Road, Huangpu District, Shanghai 200001, China. ${ }^{3}$ CAS Key Laboratory of Tissue Microenvironment and Tumor, Shanghai Institute of Nutrition and Health Shanghai Institutes for Biological Sciences, University of Chinese Academy of Sciences, Chinese Academy of Sciences, No. 320, Yueyang Road, Xuhui District, Shanghai 200001, China.

Received: 9 October 2018 Accepted: 27 December 2018

Published online: 09 January 2019

\section{References}

1. Salzman J, et al. Circular RNAs are the predominant transcript isoform from hundreds of human genes in diverse cell types. PLoS One. 2012;7(2):e30733.

2. Cocquerelle C, et al. Mis-splicing yields circular RNA molecules. FASEB J. 1993;7(1):155-60

3. Kolakofsky D. Isolation and characterization of Sendai virus DI-RNAs. Cell. 1976;8(4):547-55

4. Sanger $\mathrm{HL}$, et al. Viroids are single-stranded covalently closed circular RNA molecules existing as highly base-paired rod-like structures. Proc Natl Acad Sci U S A. 1976;73(11):3852-6.

5. Hsu MT, Coca-Prados M. Electron microscopic evidence for the circular form of RNA in the cytoplasm of eukaryotic cells. Nature. 1979;280(5720):339-40.

6. Kos A, et al. The hepatitis delta (delta) virus possesses a circular RNA. Nature. 1986;323(6088):558-60.

7. Nigro JM, et al. Scrambled exons. Cell. 1991;64(3):607-13.

8. Matsumoto Y, Fishel R, Wickner RB. Circular single-stranded RNA replicon in Saccharomyces cerevisiae. Proc Natl Acad Sci U S A. 1990;87(19):7628-32.

9. Rybak-Wolf A, et al. Circular RNAs in the mammalian brain are highly abundant, conserved, and dynamically expressed. Mol Cell. 2015;58(5):870-85.

10. Westholm JO, et al. Genome-wide analysis of drosophila circular RNAs reveals their structural and sequence properties and age-dependent neural accumulation. Cell Rep. 2014;9(5):1966-80

11. Guarnerio J, et al. Oncogenic role of fusion-circRNAs derived from Cancerassociated chromosomal translocations. Cell. 2016;166(4):1055-6.
12. Harland R, Misher L. Stability of RNA in developing Xenopus embryos and identification of a destabilizing sequence in TFIIIA messenger RNA. Development. 1988;102(4):837-52.

13. Pamudurti NR, et al. Translation of CircRNAs. Mol Cell. 2017:66(1):9-21 e7.

14. Jeck WR, et al. Circular RNAs are abundant, conserved, and associated with ALU repeats. RNA. 2013;19(2):141-57.

15. Guo JU, et al. Expanded identification and characterization of mammalian circular RNAs. Genome Biol. 2014;15(7):409.

16. Salzman J, et al. Cell-type specific features of circular RNA expression. PLoS Genet. 2013;9(9):e1003777.

17. Zheng Q, et al. Circular RNA profiling reveals an abundant circHIPK3 that regulates cell growth by sponging multiple miRNAs. Nat Commun. 2016;7:11215

18. Zhang $\mathrm{XO}$, et al. Complementary sequence-mediated exon circularization. Cell. 2014;159(1):134-47.

19. Liang D, Wilusz JE. Short intronic repeat sequences facilitate circular RNA production. Genes Dev. 2014;28(20):2233-47.

20. Ashwal-Fluss $R$, et al. circRNA biogenesis competes with pre-mRNA splicing. Mol Cell. 2014;56(1):55-66.

21. Athanasiadis A, Rich A, Maas S. Widespread A-to-l RNA editing of Alu-containing mRNAs in the human transcriptome. PLoS Biol. 2004;2(12):e391.

22. Ramaswami $\mathrm{G}$, et al. Accurate identification of human Alu and non-Alu RNA editing sites. Nat Methods. 2012;9(6):579-81.

23. Ivanov $A$, et al. Analysis of intron sequences reveals hallmarks of circular RNA biogenesis in animals. Cell Rep. 2015;10(2):170-7.

24. Li X, Yang L, Chen LL. The biogenesis, functions, and challenges of circular RNAs. Mol Cell. 2018;71(3):428-42.

25. Aktas T, et al. DHX9 suppresses RNA processing defects originating from the Alu invasion of the human genome. Nature. 2017:544(7648):115-9.

26. Errichelli $\mathrm{L}$, et al. FUS affects circular RNA expression in murine embryonic stem cell-derived motor neurons. Nat Commun. 2017:8:14741.

27. Fei $T$, et al. Genome-wide CRISPR screen identifies HNRNPL as a prostate cancer dependency regulating RNA splicing. Proc Natl Acad Sci U S A. 2017;114(26):E5207-15

28. Conn SJ, et al. The RNA binding protein quaking regulates formation of circRNAs. Cell. 2015;160(6):1125-34

29. Zhang Y, et al. Circular intronic long noncoding RNAs. Mol Cell. 2013;51(6): 792-806.

30. Li Z, et al. Exon-intron circular RNAs regulate transcription in the nucleus. Nat Struct Mol Biol. 2015;22(3):256-64.

31. Salmena $L$, et al. A ceRNA hypothesis: the Rosetta stone of a hidden RNA language? Cell. 2011;146(3):353-8.

32. Karreth FA, Pandolfi PP. ceRNA cross-talk in cancer: when ce-bling rivalries go awry. Cancer Discov. 2013;3(10):1113-21.

33. Hansen TB, et al. Natural RNA circles function as efficient microRNA sponges. Nature. 2013:495(7441):384-8.

34. Memczak $S$, et al. Circular RNAs are a large class of animal RNAs with regulatory potency. Nature. 2013;495(7441):333-8.

35. Kleaveland $B$, et al. A network of noncoding regulatory RNAs acts in the mammalian brain. Cell. 2018:174(2):350-62

36. Han D, et al. Circular RNA circMTO1 acts as the sponge of microRNA-9 to suppress hepatocellular carcinoma progression. Hepatology. 2017:66(4):1151-64.

37. Chen $\mathrm{G}$, et al. circHIPK3 regulates cell proliferation and migration by sponging miR-124 and regulating AQP3 expression in hepatocellular carcinoma. Cell Death Dis. 2018;9(2):175.

38. Li Y, et al. CircHIPK3 sponges miR-558 to suppress heparanase expression in bladder cancer cells. EMBO Rep. 2017;18(9):1646-59.

39. Zeng $\mathrm{K}$, et al. CircHIPK3 promotes colorectal cancer growth and metastasis by sponging miR-7. Cell Death Dis. 2018;9(4):417.

40. Shan $\mathrm{K}$, et al. Circular noncoding RNA HIPK3 mediates retinal vascular dysfunction in diabetes mellitus. Circulation. 2017;136(17):1629-42.

41. Ke Z, et al. CircHIPK3 promotes proliferation and invasion in nasopharyngeal carcinoma by abrogating miR-4288-induced ELF3 inhibition. J Cell Physiol. 2018

42. Jin P, et al. CircRNA circHIPK3 serves as a prognostic marker to promote glioma progression by regulating miR-654/IGF2BP3 signaling. Biochem Biophys Res Commun. 2018;503(3):1570-4.

43. Liu $X$, et al. Circular RNA HIPK3 regulates human lens epithelial cells proliferation and apoptosis by targeting the miR-193a/CRYAA axis. Biochem Biophys Res Commun. 2018:503(4):2277-85.

44. Kai D, et al. Circular RNA HIPK3 promotes gallbladder cancer cell growth by sponging microRNA-124. Biochem Biophys Res Commun. 2018;503(2):863-9. 
45. Wang J, et al. Circular RNA circHIPK3 acts as the sponge of microRNA-124 to promote human oral squamous cell carcinoma cells proliferation. Zhonghua Kou Qiang Yi Xue Za Zhi. 2018;53(8):546-51.

46. Tian F, et al. Circular RNA CircHIPK3 Promotes NCl-H1299 and NCl-H2170 Cell Proliferation through miR-379 and its Target IGF1. Zhongguo Fei Ai Za Zhi. 2017;20(7):459-67.

47. Cheng J, et al. Regulatory network of circRNA-miRNA-mRNA contributes to the histological classification and disease progression in gastric cancer. J Transl Med. 2018;16(1):216.

48. Li F, et al. Identification of the tumor-suppressive function of circular RNA ITCH in glioma cells through sponging miR-214 and promoting linear ITCH expression. Am J Transl Res. 2018;10(5):1373-86.

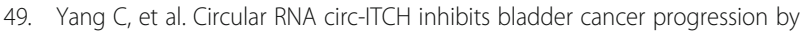
sponging miR-17/miR-224 and regulating p21, PTEN expression. Mol Cancer. 2018;17(1):19.

50. Hansen TB, et al. miRNA-dependent gene silencing involving Ago2mediated cleavage of a circular antisense RNA. EMBO J. 2011;30(21):4414-22.

51. Du WW, et al. Foxo3 circular RNA retards cell cycle progression via forming ternary complexes with p21 and CDK2. Nucleic Acids Res. 2016;44(6):2846-58.

52. Du WW, et al. Foxo3 circular RNA promotes cardiac senescence by modulating multiple factors associated with stress and senescence responses. Eur Heart J. 2017;38(18):1402-12.

53. Xia P, et al. A circular RNA protects dormant hematopoietic stem cells from DNA sensor cGAS-mediated exhaustion. Immunity. 2018;48(4):688-701 e7.

54. Abe $\mathrm{N}$, et al. Rolling circle amplification in a prokaryotic translation system using small circular RNA. Angew Chem Int Ed Engl. 2013;52(27):7004-8.

55. Abe N, et al. Rolling circle translation of circular RNA in living human cells. Sci Rep. 2015:5:16435.

56. Legnini l, et al. Circ-ZNF609 is a circular RNA that can be translated and functions in Myogenesis. Mol Cell. 2017;66(1):22-37 e9.

57. Yang $Y$, et al. Novel role of FBXW7 circular RNA in repressing glioma tumorigenesis. J Natl Cancer Inst. 2018;110(3).

58. Zhang $M$, et al. A novel protein encoded by the circular form of the SHPRH gene suppresses glioma tumorigenesis. Oncogene. 2018;37(13):1805-14.

59. Wesselhoeft RA, Kowalski PS, Anderson DG. Engineering circular RNA for potent and stable translation in eukaryotic cells. Nat Commun. 2018;9(1):2629.

60. Yang $Y$, et al. Extensive translation of circular RNAs driven by $\mathrm{N}(6)$ methyladenosine. Cell Res. 2017;27(5):626-41.

61. Glažar P, Papavasileiou P, Rajewsky N. circBase: a database for circular RNAs. RNA. 2014;20(11):1666-70.

62. Dong R, et al. CIRCpedia v2: an updated database for comprehensive circular RNA annotation and expression comparison. Genomics Proteomics Bioinformatics. 2018.

63. Zheng $L$, et al. deepBase v2.0: identification, expression, evolution and function of small RNAs, LncRNAs and circular RNAs from deep-sequencing data. Nucleic Acids Res. 2016;44(D1):D196-202

64. Chen $X$, et al. circRNADb: a comprehensive database for human circular RNAs with protein-coding annotations. Sci Rep. 2016;6:34985.

65. Liu Y, et al. CircNet: a database of circular RNAs derived from transcriptome sequencing data. Nucleic Acids Res. 2016;44(D1):D209-15.

66. Li J, et al. starBase v2.0: decoding miRNA-ceRNA, miRNA-ncRNA and protein-RNA interaction networks from large-scale CLIP-Seq data. Nucleic Acids Res. 2014;42(Database issue):D92-7.

67. Xia S, et al. CSCD: a database for cancer-specific circular RNAs. Nucleic Acids Res. 2018;46(D1):D925-9.

68. Dudekula D, et al. Circlnteractome: a web tool for exploring circular RNAs and their interacting proteins and microRNAs. RNA Biol. 2016;13(1):34-42.

69. Wu S, et al. CirclncRNAnet: an integrated web-based resource for mapping functional networks of long or circular forms of noncoding RNAs. Gigascience. 2018;7(1):1-10.

70. Li S, et al. exoRBase: a database of circRNA, IncRNA and mRNA in human blood exosomes. Nucleic Acids Res. 2018;46(D1):D106-12.

71. Zhao Z, et al. circRNA disease: a manually curated database of experimentally supported circRNA-disease associations. Cell Death Dis 2018;9(5):475.

72. Yu J, et al. Circular RNA CSMARCA5 inhibits growth and metastasis in hepatocellular carcinoma. J Hepatol. 2018;68(6):1214-27.

73. Yao Z, et al. ZKSCAN1 gene and its related circular RNA (circZKSCAN1) both inhibit hepatocellular carcinoma cell growth, migration, and invasion but through different signaling pathways. Mol Oncol. 2017;11(4):422-37.
74. Fang $L$, et al. Enhanced breast cancer progression by mutant $p 53$ is inhibited by the circular RNA circ-Ccnb1. Cell Death Differ. 2018.

75. He R, et al. CircGFRA1 and GFRA1 act as ceRNAs in triple negative breast cancer by regulating miR-34a. J Exp Clin Cancer Res. 2017;36(1):145.

76. Chen B, et al. circEPSTI1 as a prognostic marker and mediator of triplenegative breast Cancer progression. Theranostics. 2018;8(14):4003-15.

77. Dai $X$, et al. RNA-binding protein trinucleotide repeat-containing $6 \mathrm{~A}$ regulates the formation of circular RNA 0006916, with important functions in lung Cancer cells. Carcinogenesis. 2018.

78. Tan S, et al. Circular RNA F-circEA produced from EML4-ALK fusion gene as a novel liquid biopsy biomarker for non-small cell lung cancer. Cell Res. 2018;28(6):693-5.

79. Hsiao KY, et al. Noncoding effects of circular RNA CCDC66 promote Colon Cancer growth and metastasis. Cancer Res. 2017;77(9):2339-50.

80. Li X, et al. Circular RNA circlTGA7 inhibits colorectal cancer growth and metastasis by modulating the Ras pathway and upregulating transcription of its host gene ITGA7. J Pathol. 2018.

81. Militello G, et al. Screening and validation of IncRNAs and circRNAs as miRNA sponges. Brief Bioinform. 2017;18(5):780-8

\section{Ready to submit your research? Choose BMC and benefit from:}

- fast, convenient online submission

- thorough peer review by experienced researchers in your field

- rapid publication on acceptance

- support for research data, including large and complex data types

- gold Open Access which fosters wider collaboration and increased citations

- maximum visibility for your research: over $100 \mathrm{M}$ website views per year

At $\mathrm{BMC}$, research is always in progress.

Learn more biomedcentral.com/submissions 